\title{
UNA INTEGRACIÓN A SISTEMAS DE GESTIÓN DE APRENDIZAJE BASADA EN ESTÁNDARES DE UN SISTEMA BARRA-BOLA
}

\author{
A. Montoro, I. Ruano-Ruano, E. Estévez, J. Gómez Ortega, J. Gámez García \\ Escuela Politécnica Superior de Jaén. \\ Universidad de Jaén \\ Email: am100074@red.ujaen.es ,\{alonso, eestevez, juango,jggarcia\}@ujaen.es
}

\begin{abstract}
Resumen
Los laboratorios de tipo online tienen cada vez más aceptación dentro de la educación universitaria relacionada con las ciencias, tecnologías, ingenierías y matemáticas (CTIM o STEM en inglés), donde el trabajo práctico es de vital importancia para los estudiantes. El presente trabajo trata un ejemplo de integración de un laboratorio online en un sistema de gestión de aprendizaje (LMS-Learning Management System). Los estándares SCORM (Shareable Content Object Reference Model) y LTI (Learning Tool Interoperability) ofrecen la posibilidad de integrar laboratorios online en un LMS. Este trabajo, ha adaptado un sistema barra-bola convencional para posteriormente tratarlo como ejemplo de integración de un laboratorio online virtual y remoto al LMS de la Universidad de Jaén a través del estándar SCORM.
\end{abstract}

Palabras Clave: e-learning, plataforma de aprendizaje, integración de sistemas, laboratorio online, LMS, SCORM, LTI

\section{INTRODUCCIÓN}

La creación de laboratorios docentes es fundamental para la realización de trabajos prácticos en todas las áreas de enseñanza. Para los estudios universitarios STEM (Science, Technology, Engineering and Mathematics), donde el uso de laboratorios docentes es habitual, es mucho más relevante [3]. Sin embargo, la realización de este tipo de laboratorios lleva consigo un coste elevado, cuyo origen suele ser la puesta en marcha y el mantenimiento, además de ser imprescindible el uso de un espacio físico. Este último condicionante, se ha visto agravado por la situación de pandemia mundial vivida durante el año pasado, que imposibilita el uso de los espacios universitarios por parte de los alumnos. Gracias a ello, se ha podido demostrar y ratificar las amplias ventajas de los laboratorios online dentro de la enseñanza. [4], [7].

Dentro de las Tecnologías de la Información y las Comunicaciones (TIC), uno de los elementos TIC más importantes para la docencia serían las plataformas de docencia o LMS (Learning Management System). Los LMS, que se encuentran instaurados en las instituciones universitarias y a los cuales los estudiantes se encuentran habituados, suelen ser denominados también como entornos de aprendizaje virtual o VLE (Virtual Learning Environment).

En el entorno tecnológico, la finalidad de un estándar es implantar normas que permitan el entendimiento y la colaboración entre los diferentes sistemas.

El uso de estándares como SCORM (Shareable Content Object Reference Model) y LTI (Learning Tool Interoperability) se ha utilizado en diferentes trabajos para la integración de este tipo de laboratorios online y LMS, y su uso tiene muchas ventajas. [1]

Debido al uso del LTI los estudiantes pueden acceder a un laboratorio online, que se encuentra alojado en un servidor externo al LMS, como si estuviera en el mismo LMS. Por otro lado, mediante el uso de SCORM los estudiantes pueden acceder a un laboratorio online que se encuentra alojado en el mismo LMS, embebido en el interior de un paquete SCORM. En ambos casos los estudiantes deben identificarse en el LMS, con LTI acceden al laboratorio al iniciar un elemento enlazado con la aplicación del laboratorio, mientras que con SCORM acceden a la aplicación del laboratorio al iniciar un paquete SCORM.

El presente trabajo se centra precisamente en facilitar unas pautas para la integración de un laboratorio a un LMS a través de dichos estándares. Por ello, el segundo apartado presenta una idea general sobre los estándares LTI y SCORM. En el tercero se indican los pasos a seguir para integrar un laboratorio a través de los estándares anteriormente mencionados. El aparatado 4 se centra en caracterizar y adaptar un sistema barra-bola para posteriormente, en el apartado 5, describir cómo se produce su integración en el LMS de la UJA usando LTI y SCORM. Finalmente, se incluye un apartado de Conclusiones.

\section{ESTÁNDARES LTI Y SCORM.}

Como anteriormente se ha mencionado los estándares son la manera de establecer unas normas para el entendimiento y la colaboración entre sistemas. Dentro de la comunidad e-learning también tienen esa propiedad de establecer normas, pero relacionados con sistemas educativos, y se pueden clasificar según categorías como serían: Accesibilidad, Arquitectura, Calidad, Competencias, Contenidos y Evaluación, 
Derechos digitales, Información del alumno, Interactividad, Metadatos; Proceso de Aprendizaje, Repositorios y Vocabulario y Lenguajes. [2]

Los estándares se encuentran ligados con la interactividad $y$ arquitectura que facilitan la integración de contenidos de carácter educativo como son los laboratorios con las plataformas de aprendizaje. Los más extendidos y habituales son los denominados LTI y SCORM.

A continuación, en los siguientes sub-apartados se va a detallar el carácter funcional de los estándares LTI y SCORM.

\section{$2.1 \quad$ SCORM}

Tiene origen en la secretaría de defensa de los Estados Unidos de América y fue creado por Advanced Distributed Learning (ADL).

El estándar SCORM tiene varias versiones, la última de ellas es la versión SCORM 2004 4th edition.

SCORM introduce pautas para crear y empaquetar contenido de tipo e-learning, con la posibilidad de comunicación entre el contenido y el LMS (por ejemplo, ILIAS o Moodle) del que se encuentre instalado el paquete para el intercambio de datos y, por último, las indicaciones para realizar la secuenciación y navegación entre los componentes educativos que se encuentran incluidos.

La última versión tiene 3 sub-especificaciones:

- CAM (Content Aggregation Model). Determina cómo describir la estructura de un elemento educativo, en base a componentes más pequeños, y la forma de empaquetar ese contenido para después se pueda ejecutar en LMS.

- RTE (Run-Time Environment). Determina cómo efectuar el contenido, la comunicación con el LMS y cómo realizar el seguimiento del progreso que estaría realizando el alumno.

- SN (Sequencing and Navigation). Determina como definir la secuencia de ejecución y la navegación entre componentes de un paquete SCORM.

El uso de SCORM no es algo novedoso, desde 2007 se han realizado trabajos integrándolos en LMS [6], [12], sin embargo, la implementación de estos trabajos de forma real no se obtuvo hasta años más tarde. [8][10]. En ellos, si se tenía un uso más profundo de SCORM RTE, utilizando el intercambio de datos entre LMS y el laboratorio [11], [15], ya que, cuando la integración es más avanzada el intercambio de datos de aprendizaje con la base del LMS es realizada por el propio software.

\section{$2.2 \quad$ LTI}

Este estándar está desarrollado por el IMS Global Learning Consortium (IMS) [13], su objetivo es la integración entre aplicaciones de aprendizaje con plataformas de aprendizaje. Las aplicaciones o herramientas se encuentran alojadas de modo remoto en las plataformas de aprendizaje, como podrían ser LMSs o cualquier otro tipo de entorno.

Los tres términos que utiliza LTI son:

- Tool: referente a la aplicación de aprendizaje enriquecida

- Tool Provider: referente a los sistemas que proporcionan las herramientas.

- Tool Consumer: referente a los sistemas que consumen las herramientas que proporcionan los Tool Providers, por ejemplo, LMSs aunque podría ser otro tipo de plataforma de aprendizaje e-learning.

Habitualmente, el uso que se le da al LTI suele ser que un Tool Consumer ofrece a un usuario contenidos externos con apariencia de estar alojados en el LMS, pero el usuario en realidad cuando accede a un elemento LTI del LMS, es el LMS que usa el estándar LTI para lanzar la Tool ubicada de forma externa en el Tool Provider. Se produce por tanto un intercambio de datos entre el LMS y la Tool utilizada.

LTI al ser tan popular y conocido, se pueden encontrar muchas plataformas e-learning que lo utilizan, o bien con el rol de consumidoras (Tool Consumer), o bien con el rol de ser consumidas (Tool Provider).

Al igual que ocurre con SCORM existen varias versiones de LTI, pero algunas versiones son más conocidas que otras. De hecho, la recomendación de IMS (el desarrollador de LTI) es saltar las versiones 1.1.1, 1.2 y 2.0, pasando así de la versión 1.1 a la 1.3.

\section{PAUTAS PARA INTEGRAR LABORATORIOS ONLINE USANDO ESTÁNDARES LTI Y SCORM}

Existen diferentes estándares que pueden ayudar a alcanzar la integración de un laboratorio online y un LMS. En este trabajo se han tratado los 2 estándares más utilizados y conocidos para este fin, LTI y SCORM.

\subsection{INTEGRACIÓN CON LTI}

La integración de un laboratorio online y un LMS usando el estándar LTI se puede alcanzar de 3 modos diferentes que se explicarán a continuación.

I. NATIVA. Es el modo más natural e intuitivo de realizar la integración entre el laboratorio online y el LMS. En este modo el LMS actúa como un consumidor de herramientas LTI, siendo la herramienta una aplicación de laboratorio online 
ubicada en un servidor externo al LMS (proveedor de herramientas LTI).

El usuario realiza su conexión con el LMS donde se tiene alojado el elemento LTI que al ser iniciado ejecuta un laboratorio virtual que se encuentra alojado en un servidor externo que aparece en la pantalla del usuario.

Si además de ser un laboratorio online es remoto la forma de acceder al servidor es un poco diferente, pues el software del laboratorio LTI debe de tener acceso al sistema real (maqueta), que podría ser el mismo servidor u otro externo.

II. INVERSA. Es una forma menos intuitiva de realizar la integración entre un laboratorio online y un LMS. En este modo el laboratorio actúa como consumidor de recursos de un LMS mediante el uso del estándar LTI.

Los recursos de un LMS que se pueden consumir desde la aplicación del laboratorio pueden ser muy variados, por ejemplo, un test del LMS que sirva para evaluar los conocimientos del usuario que entra en el laboratorio o una encuesta de valoración del laboratorio (ambos tipos de recursos pueden usarse antes y/o después de trabajar en el laboratorio).

III. MIXTA. Este modo combina los dos modos anteriores de forma que se produce una integración en 2 sentidos: de forma nativa e inversa. En este caso, el usuario se identifica en el LMS y al iniciar un objeto LTI se produce la ejecución de la aplicación del laboratorio online, que se ubica en un servidor externo. Hasta aquí coincide con la integración nativa. Adicionalmente, la aplicación de laboratorio actúa como enlace LTI de forma que inicia recursos ubicados en otro sistema (LMS) que son ofrecidos al estudiante desde el software del laboratorio.

\subsection{INTEGRACIÓN CON SCORM}

La integración de un laboratorio online en un LMS utilizando el estándar SCORM se consigue editando un paquete SCORM. El software del laboratorio debe incluirse dentro de uno de sus componentes con capacidad de usar el modelo de datos de SCORM y comunicarse con el LMS (SCO o Sharable Content Object).

Los SCOs se implementan como páginas Web, por lo que la aplicación del laboratorio debe estar en el interior de la página web del SCO y haber sido programada usando una tecnología compatible.

Para lograr una integración avanzada laboratorio-LMS la aplicación del laboratorio debe usar las funciones JavaScript de la sub-especificación RTE de SCORM. Con estas funciones se pueden obtener y manipular los datos del modelo SCORM, aunque es recomendable usar alguna librería que facilite este trabajo [10].

\section{SISTEMA BARRA-BOLA}

Se ha trabajado en un sistema barra-bola que se ha caracterizado y adaptado para ofrecerse como laboratorio online en dos versiones: virtual y remota.

Este tipo de laboratorio es de gran utilidad para los Grados de Ingeniería Electrónica Industrial, en aquellas asignaturas que tienen relación con el control automático.

El sistema barra-bola está compuesto por tres módulos principales: el motor, la barra y la bola tal y como ilustra la Figura 1.

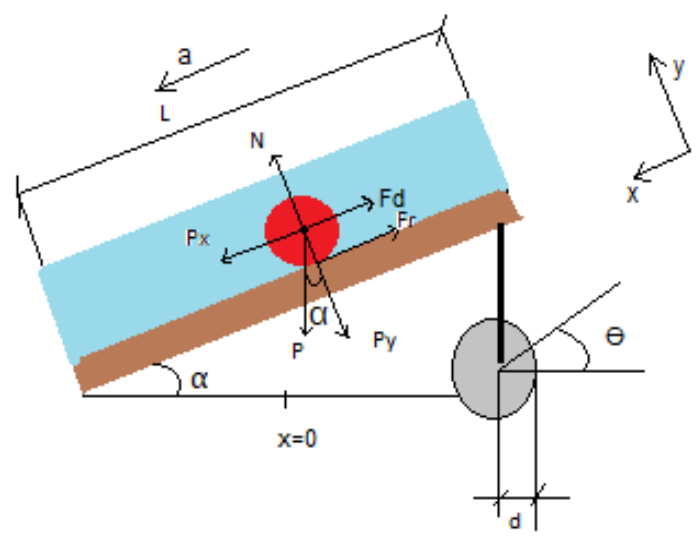

Figura 1. Sistema barra-bola

La bola se encuentra situada encima de la barra. La función de este sistema es mover la bola sobre la barra. Para ello, el motor rota un ángulo, $\Theta, y$, por consecuencia, la barra se inclina un ángulo, $\alpha$, que termina generando el movimiento de la bola sobre la barra.

Debido a que este sistema de barra-bola iba a ser implementado como un laboratorio online remoto se han introducido unas modificaciones para mejorar su funcionamiento en remoto.

Unas de las modificaciones es transformar la barra en un tubo de metacrilato donde se introducirá la bola y hará dentro del sistema el papel de barra, con la ventaja de que si la bola está dentro del tubo jamás podría caerse fuera. Esto es una gran ventaja porque al ser un laboratorio online remoto no tendría que tener una persona vigilando que el sistema esté funcionando correctamente $\mathrm{y}$, por consecuencia, el mantenimiento es menor.

La segunda modificación sería introducir dentro del tubo de metacrilato, junto con la bola, una mezcla con un cierto porcentaje de glicerina con agua. De esta forma el sistema se ralentizar y será mucho más visual y no tan brusco su movimiento. 
Antes de realizar el laboratorio online remoto, al principio se realizó un laboratorio virtual. Para ello se hizo uso del software de Easy Java/JavaScript Simulations (EJSS) [5] para crear la simulación de control del sistema barra-bola. Este laboratorio se muestra en la Figura 2.

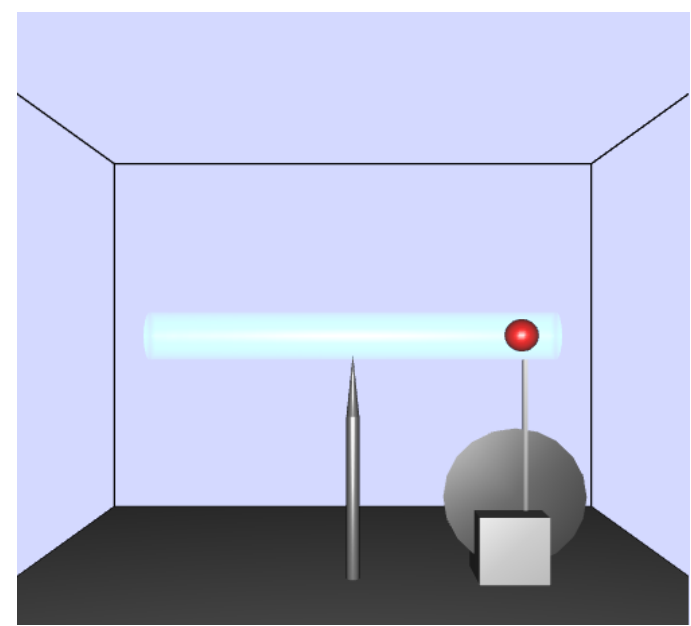

Figura 2. Laboratorio virtual (sistema barra-bola)

Las constantes del motor en corriente continua, tanto $\mathrm{Km}$, ganancia estática y Tm, constante de tiempo, se han personalizado de forma que según el estudiante que acceda al laboratorio tendrán unas constantes distintas. Al igual ocurre con el porcentaje de glicerina que se ha mencionado anteriormente que también era un parámetro personalizado.

Otra función destacable es la evaluación automática que se realiza del estudiante a medida que se introducen los resultados en los apartados de evaluación. Y se muestra al estudiante su calificación.

La Figura 3 muestra una imagen de la maqueta del sistema Barra-Bola.

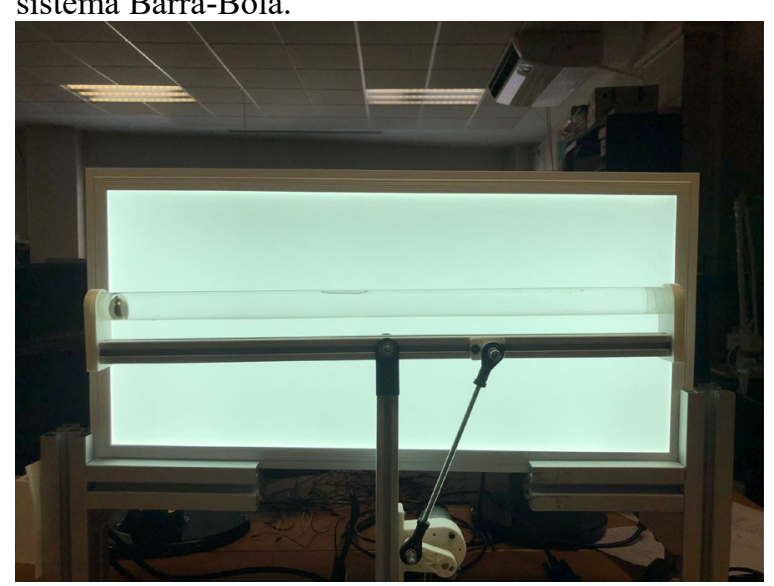

Figura 3. Maqueta del sistema barra-bola

La segunda modificación realizada en el laboratorio remoto, en la que se introduce la mezcla de glicerina con agua, se utiliza en el laboratorio virtual para crear un parámetro personalizado según el estudiante. Por ello, cada estudiante tendría un sistema barra-bola personalizado sobre el que realizar su experimento.

Para el sistema real (maqueta) se han utilizado los siguientes componentes:

- Actuador: Un motor en corriente continua con escobillas capaz de funcionar a $12 \mathrm{~V}$ que contiene una caja de engranajes con relación 70:1 (Figura 4). Esta caja de engranajes está compuesta principalmente de engranajes rectos, pero en la primera etapa cuenta con engranajes helicoidales que reduce el ruido y lo vuelve más eficiente.

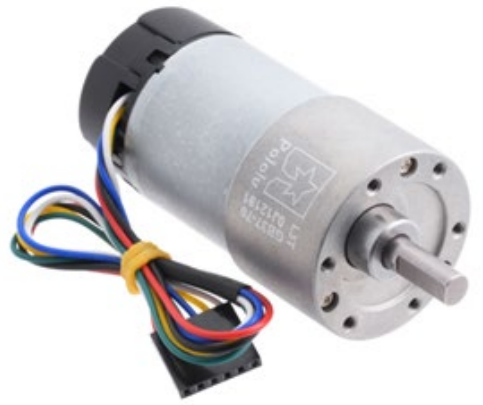

Figura 4. Motor en corriente continua

Al motor se le acopla un puente en $\mathrm{H}$ por el cual se realiza el movimiento de giro hacia derecha e izquierda del motor (Figura 5).

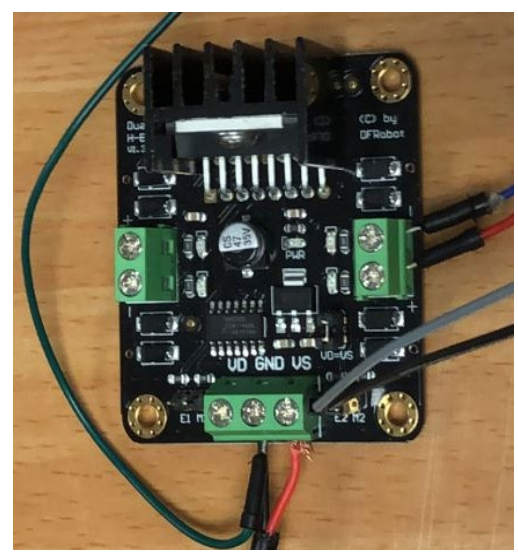

Figura 5. Puente H

- Planta: Formada por la barra y la bola.

- Sensor de posición: Se trata de un sensor virtual creado por software por el cual mediante visión se calcula la distancia desde el centro de la bola al centro de la barra siguiendo el diagrama de la Figura 6.

Este sistema podría ser utilizado para crear ejemplos prácticos de control. Por ejemplo, un control en posición de la bola o el control en velocidad de la bola. En el laboratorio remoto se ha adaptado el software desarrollado con EJsS para la versión virtual para obtener el programa cliente que usarán los estudiantes para poder realizar los experimentos remotos en la 
planta real. Para la comunicación entre este software y la planta real se ha usado el protocolo RIP (Remote Interoperability Protocol) [14].

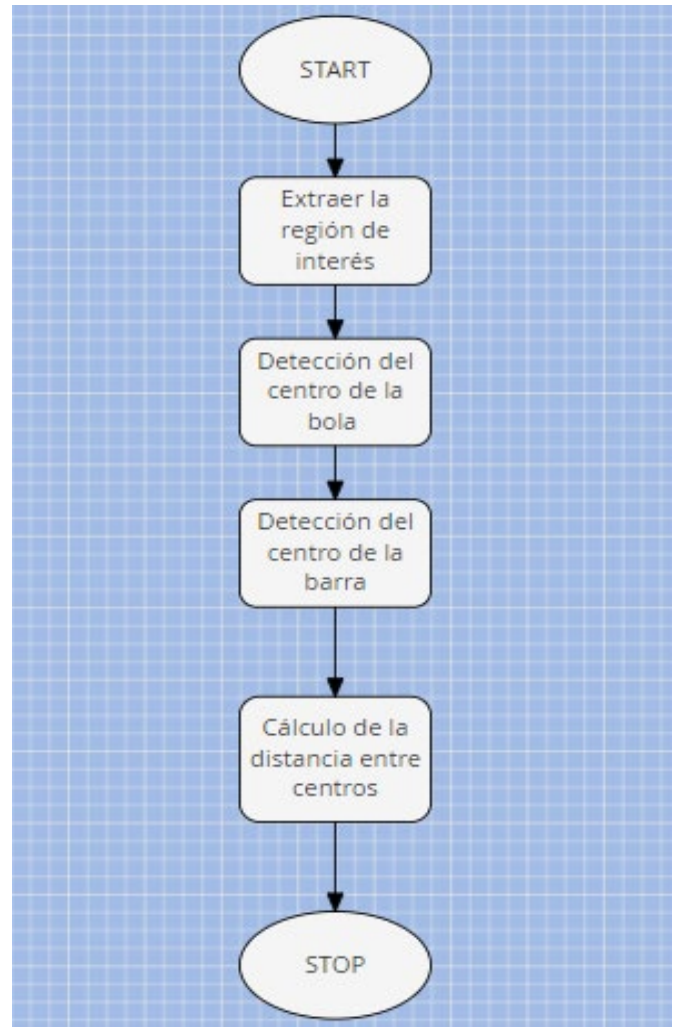

Figura 6. Diagrama de flujo del software de medida por visión.

\section{INTEGRACIÓN DEL SISTEMA BARRA BOLA EN EL LMS DE LA UJA CON ESTÁNDARES}

El desarrollo de los laboratorios online del sistema barra-bola (virtual y remoto) se han realizado dentro del proyecto UniLabs (University Network of Interactive Laboratories, https://unilabs.dia.uned.es/). En este proyecto están involucradas 14 universidades españolas, entre las que se encuentra la UJA, y está siendo dirigido por la UNED.

La integración de los laboratorios se va a llevar a cabo usando los dos estándares tratados en este trabajo. Los siguientes sub-apartados presentan brevemente los pasos a seguir.

\subsection{INTEGRACIÓN CON LTI}

El software EJsS con el que se desarrolló la aplicación de laboratorio que usa el estudiante, tanto para el laboratorio virtual como para el laboratorio remoto, permite generar un fichero comprimido ZIP que incluye toda la estructura necesaria.
Este fichero se ubica en un repositorio externo que se comporta como el proveedor de herramientas de LTI, en este caso el LMS de la UNED que alberga el proyecto UNILabs. Se trata de un sistema Moodle en el que se sube el fichero del laboratorio y configura como accesible a través del estándar LTI.

El LMS de la UJA actúa como un consumidor de herramientas; en una ubicación accesible por los estudiantes se crea un elemento LTI que se enlaza con el laboratorio accesible por LTI ubicado en el LMS de UniLabs.

De esta forma los estudiantes de la UJA acceden a su LMS para trabajar con el laboratorio ubicado en el LMS de UniLabs.

\subsection{INTEGRACIÓN CON SCORM}

El mismo software de EJsS, también en este caso para la modalidad virtual y remota, ofrece la posibilidad de exportarlo como paquete SCORM. Este paquete ya podría ser importado en cualquier LMS compatible. Desde el LMS de la UJA debe importarse el fichero ZIP para crear un paquete SCORM en una ubicación accesible por el alumno. Este módulo SCORM incluye en su interior la aplicación del laboratorio (virtual o remoto).

Antes de realizar la exportación en EJsS se tiene la posibilidad de adaptar el código a SCORM-RTE para poder realizar el intercambio de datos entre LMS y la simulación.

\section{CONCLUSIONES}

Está demostrada la importancia de realizar prácticas dentro de la educación universitaria relacionada con las ciencias, tecnologías, ingenierías y matemáticas, para poder asimilar los conceptos teóricos. En este sentido, cada vez están siendo más aceptados el uso de los laboratorios online. Además, la situación y alarma social vivida en estos dos últimos cursos académicos debido al COVID-19 ha obligado a docentes a agudizar el ingenio para que el alumnado pudiera asimilar los conceptos a través de dichos laboratorios. El presente trabajo ha presentado las pautas para conseguir la integración de un laboratorio online en el correspondiente sistema de gestión de aprendizaje a través de los estándares más expandidos como son LTI y SCORM. Para ello, se ha tomado como ejemplo de laboratorio un sistema convencional barra-bola, se ha condicionado y posteriormente se ha en el LMS utilizado en la UJA a través de LTI y SCORM.

\section{Agradecimientos}

Los autores quieren agradecer la subvención parcial de este trabajo a través de los proyectos PIMED01_201921, PID2019-110291RB-I00. 


\section{English Summary}

\section{AN INTEGRATION TO LEARNING MANAGEMENT SYSTEMS BASED ON STANDARDS OF A BALL AND BEAM SYSTEM}

\begin{abstract}
Online labs are increasingly accepted within science, technology, engineering and mathematics (STEM) college education, where practical work is vitally important to students. This paper deals with an example of integrating an online laboratory into a Learning Management System (LMS). For the integration of these, the use of standards is essential. The SCORM (Shareable Content Object Reference Model) and LTI (Learning Tool Interoperability) standards offer the possibility of integrating online laboratories in an LMS (Learning Management System). For this paper, as an example of integration, the virtual and remote online laboratory, the classic bar-ball system is used.
\end{abstract}

Keywords: e-learning, learning platform, systems integration, online laboratory, Learning Tool Interoperability (LTI), Learning Management Systems (LMS).

\section{Referencias}

[1] Advanced Distributed Learning (ADL), "SCORM 2004 4th Ed. (Shared Content Object reference model)", 2009. [Online]. Available: https://www.adlnet.gov/adlresearch/scorm/scorm-2004-4th-edition/. [Accessed: 21-Jun-2016].

[2] A. Berlanga Reyes, B. Fernández Manjón, I. Martínez Ortiz, P. Moreno Ger, and J. L. Sierra Rodríguez, "Estándares en e-learning y diseño educativo", 2011.

[3] M.P. Clough, "Using the laboratory to enhance student learning" Learn. Sci. Sci. Learn., no. JANUARY 2002, pp. 85-94, 2002.

[4] J.E. Corter, J. V. Nickerson, S.K. Esche, C. Chassapis, S. Im, and J. Ma, "Constructing reality: A Study of Remote, Hands-On, and Simulated Laboratories" ACM Trans. Comput. Interact., vol. 14, no. 2, pp. 7-es, Aug. 2007.

[5] F. Esquembre, "Easy Java Simulations: a software tool to create scientific simulations in Java", Comput. Phys. Commun., vol. 156, no. 2, pp. 199-204, Jan. 2004.
[6] L. Gomes and S. Bogosyan, "Current Trends in Remote Laboratories", Ind. Electron. IEEE Trans., vol. 56, no. 12, pp. 4744-4756, 2009.

[7] J. Ma and J. V. Nickerson, "Hands-on, simulated, and remote laboratories: A Comparative Literature Review" ACM Comput. Surv., vol. 38, no. 3, pp. 7-es, Sep. 2006.

[8] V. Mateos, A. Gallardo, and T. Richter, "LiLa Booking System: Architecture and Conceptual Model of a Rig Booking System for On-Line Laboratories” ,Int. J. Online Eng., vol. 7, no. 4, 2011.

[9] T. Richter, Y. Tetour, and D. Boehringer, "Library of Labs - A European Project on the Dissemination of Remote Experiments and Virtual Laboratories", in 2011 IEEE International Symposium on Multimedia, 2011, pp. 543-548.

[10] I. Ruano, P. Cano, J. Gamez, and J. Gomez, "Advanced LMS Integration of SCORM Web Laboratories", IEEE Access, vol. 4, pp. 63526363, 2016.

[11] I. Ruano-Ruano, J. Gamez, S. Dormido, and J. Gomez, "A Methodology to Obtain Learning Effective Laboratories with Learning Management System Integration", IEEE Trans. Learn. Technol., vol. 9, no. 4, pp. 391-399, 2016.

[12] E. Sancristobal Ruiz, M. Castro, J. Harward, P. Baley, K. DeLong, and J. Hardison, "Integration view of Web Labs and Learning Management Systems", in IEEE EDUCON 2010 Conference, 2010, vol. 81, pp. 1409-1417.

[13]F. Schauer et al., "REMLABNET IV - LTI Federated Remote Laboratory Management System with Embedded Multiparameter Simulations", Int. J. Online Eng., vol. 13, no. 10, p. 103, Nov. 2017.

[14] L. de la Torre, J. Chacon, and D. Chaos, "Remote Interoperability Protocol for online laboratories" Universidad Nacional de Educacion a Distancia (UNED), ETS. Ingenieria Informatica, Madrid (Spain), 2019.

[15]H. Wuttke, M. Hamann, and K. Henke, "Integration of remote and virtual laboratories in the educational process", in Proceedings of 2015 12th International Conference on Remote Engineering and Virtual Instrumentation (REV), 2015, vol. 11, no. February, pp. 157-162.

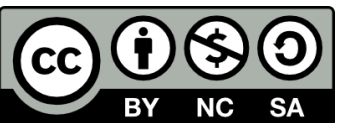
(C) 2021 by the authors. Submitted for possible open access publication under the terms and conditions of the Creative Commons Attribution CC BY-NC-SA 4.0 license (https://creativecommons.org/licenses/byncsa/4.0/deed.es). 